
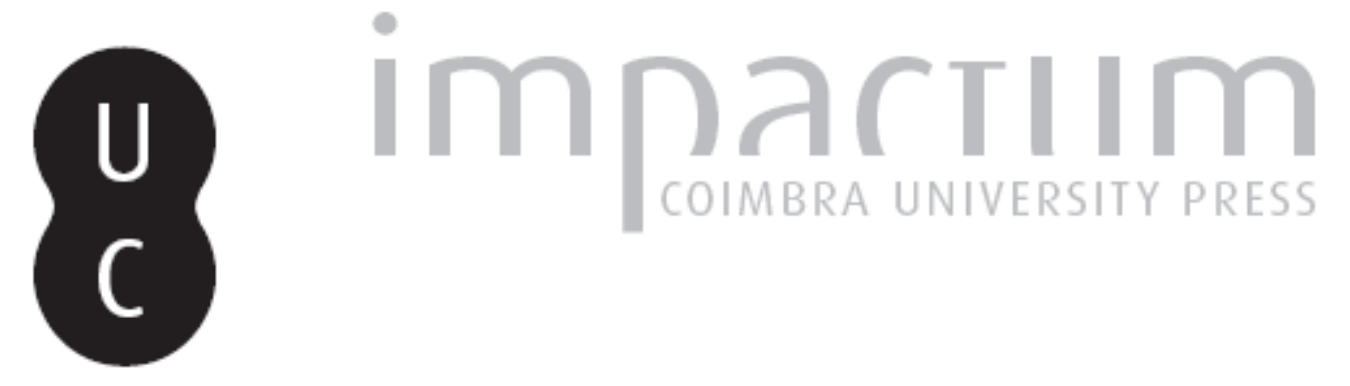

\title{
Si può parlare di un'evoluzione della dialettica platonica?
}

\section{Autor(es): Berti, Enrico}

Publicado por: Imprensa da Universidade de Coimbra

URL persistente:

URI:http://hdl.handle.net/10316.2/42267

DOI:

DOI:https://doi.org/10.14195/2183-4105_2_1

Accessed : $\quad$ 26-Apr-2023 00:16:00

A navegação consulta e descarregamento dos títulos inseridos nas Bibliotecas Digitais UC Digitalis, UC Pombalina e UC Impactum, pressupõem a aceitação plena e sem reservas dos Termos e Condições de Uso destas Bibliotecas Digitais, disponíveis em https://digitalis.uc.pt/pt-pt/termos.

Conforme exposto nos referidos Termos e Condições de Uso, o descarregamento de títulos de acesso restrito requer uma licença válida de autorização devendo o utilizador aceder ao(s) documento(s) a partir de um endereço de IP da instituição detentora da supramencionada licença.

Ao utilizador é apenas permitido o descarregamento para uso pessoal, pelo que o emprego do(s) título(s) descarregado(s) para outro fim, designadamente comercial, carece de autorização do respetivo autor ou editor da obra.

Na medida em que todas as obras da UC Digitalis se encontram protegidas pelo Código do Direito de Autor e Direitos Conexos e demais legislação aplicável, toda a cópia, parcial ou total, deste documento, nos casos em que é legalmente admitida, deverá conter ou fazer-se acompanhar por este aviso.

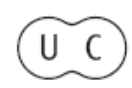




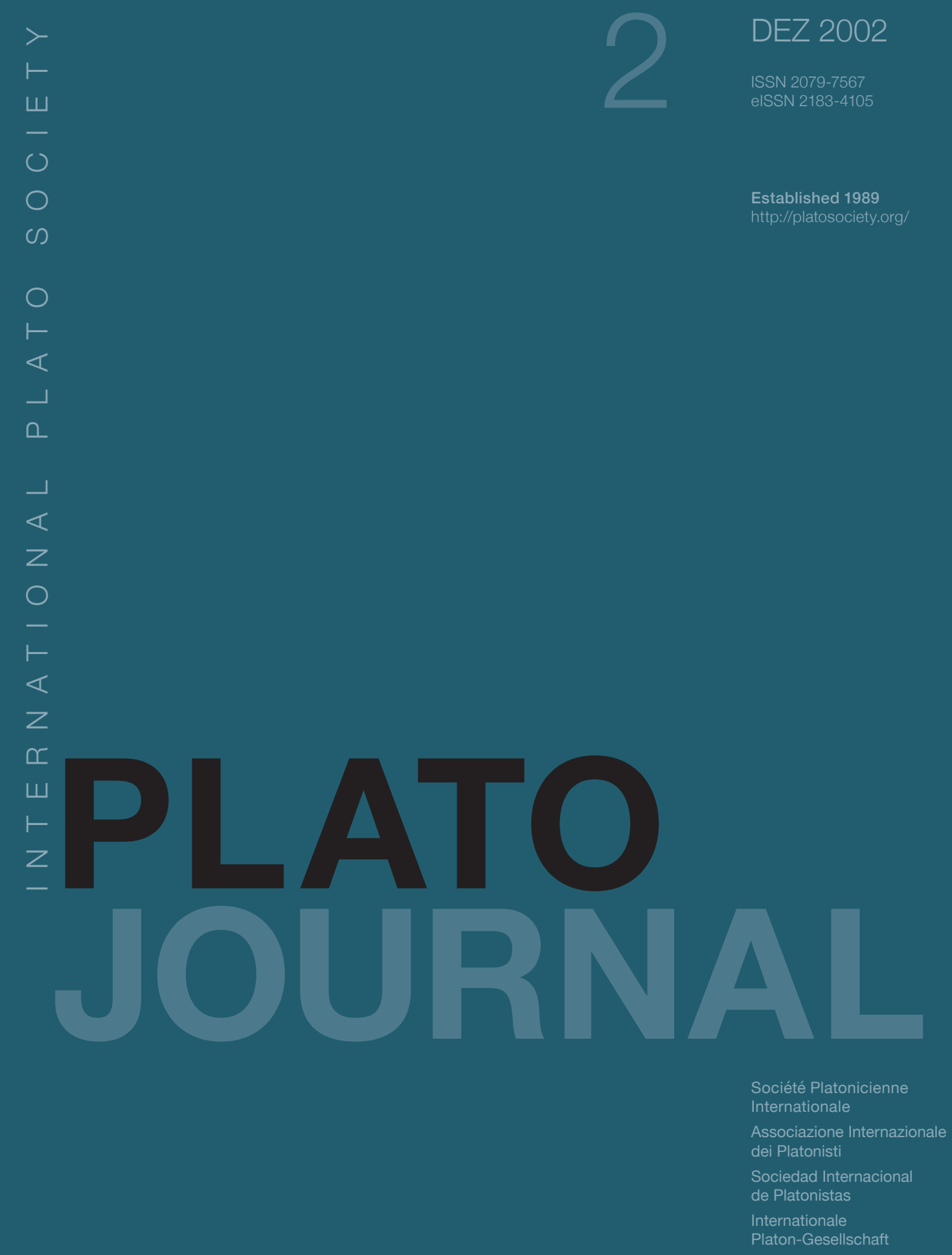




\title{
Si può parlare di un'evoluzione della dialettica platonica?
}

\author{
BERTI, Enrico, in 2. Plato 2 (2002), [En
}

ligne], January 2002

Secondo un'opinione ancora oggi diffusa, la concezione della dialettica propria di Platone avrebbe subito un'evoluzione non solo tra i dialoghi giovanili, in cui presumibilmente sarebbe contenuto il pensiero di Socrate, e i dialoghi che più propriamente esprimono il pensiero di Platone, ma anche all'interno di questi ultimi.

I più illustri responsabili di questa interpretazione sono forse Julius Stenzel e Richard Robinson. Il primo, nelle sue famose Studien zur Entwicklung der platonischen Dialektik von Sokrates zu Aristoteles (2. erw. Aufl. Leipzig und Berlin 1931), distingue lo sviluppo della dialettica platonica in due grandi periodi, espressi sinteticamente nel binomioAretê und Diairesis. Nel primo periodo, comprendente i dialoghi giovanili e quelli della maturità fino alla Repubblica, la dialettica sarebbe concepita essenzialmente come ricerca dell'aretê, intesa come causa, del doppio senso di fondamento e di fine. Nel secondo periodo, comprendente tutti i dialoghi successivi, specialmente Fedro, Sofista, Politico e Filebo, la dialettica sarebbe concepita invece come procedimento di divisione, mirante a isolare il concetto.

Robinson, nel suo altrettanto famoso libro Plato's Earlier Dialectic (second edition Oxford 1953), si limita a studiare la dialettica nei dialoghi socratici e in quelli del "periodo di mezzo" (Menone, Fedone, Repubblica, aggiungendovi nella seconda edizione anche il Parmenide), ma già con questa limitazione, e con l'indicazione di tale dialettica comeearlier, egli suggerisce che nei dialoghi successivi sia presente un diverso concetto di dialettica. Inoltre egli distingue chiaramente la concezione socratica della dialettica come elenchos, presente nei dialoghi giovanili di Platone, da quella della dialettica come metodo di discussione delle ipotesi, presente nei dialoghi del periodo di mezzo fino, appunto, al Parmenide. Il mio problema è di vedere se effettivamente si possa parlare di un'evoluzione della concezione platonica della dialettica, o se invece, pur nel prevalere di aspetti diversi, cioè l'elenchos, la discussione delle ipotesi, la diairesis, non si debba riconoscere all'interno di essa una profonda continuità, che va dai primi dialoghi almeno sino almeno alla Lettera VII.

\section{La dialettica come "elenchos" nei dialoghi giovanili}

Secondo un'opinione ormai quasi unanimemente condivisa, considero dialoghi giovanili, espressione della fase ancora "socratica" del pensiero di Platone, Eutifrone, Ippia maggiore, Carmide, Lachete, Liside e I libro della Repubblica. In questi dialoghi non è presente il termine "dialettica", ma è chiaramente praticato un procedimento, il tipico dialogare socratico (dialegesthai, da cui deriverà poi l'aggettivo dialektikê), che consiste nel porre una domanda, in genere la richiesta di definire una virtù, e nel mettere alla prova le diverse risposte che ad essa vengono date, concludendo in genere in modo negativo, cioè che nessuna di tali risposte è adeguata. Nell'Eutifrone Socrate domanda che cos'è la santità, nell'Ippia maggiore che cos'è la bellezza, nel Carmide che cos'è la saggezza, nel Lachete che cos'è il coraggio, nel Liside che cos'è l'amicizia e nel I libro della Repubblica che cos'è la giustizia. Il "mettere alla prova" le risposte che vengono date a queste domande è indicato, anche in altri dialoghi, a volte come exetasis, a volte come peira (Apol. 38a; Gorg. 448a, 515 b; Prot. 348a), ma soprattutto come elenchos. Con quest'ultimo termine si indicava nel linguaggio comune, e dunque anche in quello di Socrate e di Platone, appunto l'esaminare, il mettere alla prova, esattamente come con exetasis e peira, e quindi anche il rendere conto di qualche affermazione, il cercarne una ragione, ma con esito alterno, cioè con la possibilità sia di giustificare l'affermazione in questione, cioè di mostrarne la fondatezza, ovvero di dimostrarla, sia di smentirla, di mostrarne l'infondatezza, cioè di confutarla (cfr. Apol. 18d, 39c; Gorg. 470c).

Quest'ultimo significato è quello che si applica più frequentemente, nei dialoghi giovanili, all'operazione per mezzo della quale Socrate esamina il valore di una risposta, e pertanto fa sì che l'elenchos diventi una specie di termine tecnico con cui designare quella che potremmo chiamare la dialettica socratica. Innumerevoli sono, in questi dialoghi, gli esempi di elenchos. Robinson, che li ha studiati a fondo dal punto di vista della loro struttura logica, ha ritenuto di poterne dare la seguente definizione: "elenco nel senso più ampio significa esaminare una persona con riguardo ad un'affermazione che essa ha fatto, ponendole domande che richiedono ulteriori affermazioni, nella speranza che essa voglia determinare il significato e il valore della sua prima affermazione. Il più delle volte il valore di verità atteso è la falsità; e così l'elenco nel senso più stretto è una forma di confutazione. In questo senso esso è l'aspetto più caratteristico del comportamento di Socrate nei primi dialoghi platonici" (p. 7). 
possono valere anche per tutti gli altri. Alla domanda di Socrate "che cos'è il santo?" Eutifrone risponde anzitutto che "santo è ciò che è caro agli dèi". Allora Socrate gli pone una serie di altre domande, alle quali Eutifrone è costretto a rispondere sempre affermativamente, cioè: il santo e il non santo sono l'uno l'opposto dell'altro; gli dèi sono in lite fra loro; essi dissentono su questioni come il giusto e l'ingiusto, il bello e il brutto, il buono e il cattivo; essi amano le azioni che giudicano buone, belle e giuste, e odiano quelle che giudicano cattive, brutte e ingiuste. Da tutte queste risposte Socrate deduce che, allora, le stesse azioni possono essere care agli dèi e odiose agli dèi, e dunque, se è vera la prima risposta di Eutifrone, le stesse azioni saranno sante e non sante, mentre lo stesso Eutifrone aveva riconosciuto che il santo e il non santo sono l'uno l'opposto dell'altro (Euthyphr. 6e-8a). Socrate, insomma, deduce dalle risposte di Eutifrone che questi è in contraddizione con se stesso e che pertanto la sua prima risposta deve essere considerata falsa.

Questo procedimento corrisponde perfettamente a quello che Aristotele descriverà come sillogismo dialettico, cioè come deduzione a partire da premesse endossali (endoxa) (Top. I 1), più precisamente a quel particolare sillogismo dialettico che è la confutazione, definita da Aristotele come "deduzione della contraddizione" (An. Pr. II 20). Le risposte date da Eutifrone successivamente alla prima sono infatti endossali, cioè condivisibili da tutti, ed è questo il motivo per cui Eutifrone è costretto a concederle. Proprio lui, che era sacerdote e conosceva bene quella specie di testi sacri che erano per i Greci i poemi omerici, sapeva bene quanto e su che cosa gli dèi fossero in lite fra loro, quindi non poteva rifiutarsi di ammetterlo. Naturalmente il riconoscimento, da parte sua, della contraddizione in cui la sua prima risposta è incorsa, fa sì che lo stesso Eutifrone, d'accordo con Socrate, la lasci cadere, cioè la consideri confutata. Evidentemente tanto Socrate quanto Eutifrone, a differenza per esempio dal sofista Protagora, ritengono che la contraddizione sia segno di falsità, cioè ammettono implicitamente quello che con Aristotele diverrà il "principio di non-contraddizione" (mi permetto di rinviare, a questo proposito, al mio libro Contraddizione e dialettica negli antichi e nei moderni, Palermo 1987).

\section{Ipotesi e confutazione nei dialoghi di mezzo}

Consideriamo "dialoghi di mezzo" Menone, Fedone, Repubblica e Parmenide, la cui vicinanza anche cronologica alla Repubblica è stata dimostrata con argomenti stilometrici da L. Brandwood (A Word Index to Plato, Leeds 1976). Questi dialoghi sono caratterizzati anzitutto dal fatto che in essi compare il termine "dialettica". Nel Menone Platone distingue il modo di discutere e di confutare dei Sofisti, definiti eristici ed agonistici, da quello che si usa tra "amici" e che è definito "più dialettico" (dialektikôteron): quest'ultimo consiste, per chi risponde, nel dare risposte vere, e per chi interroga, nell'assumere come premesse solo ciò che l'interlocutore ha effettivamente concesso (75d). Si tratta dunque esattamente dello stesso procedimento praticato nei dialoghi giovanili, cioè socratici. Nel Fedone la dialettica è considerata una vera e propria arte, cioè l'arte concernente i discorsi (hê peri tous logous technê, 90b). Nella Repubblica si parla esplicitamente di un "metodo (cioè di una via, di un percorso) dialettico (hê dialektikê methodos, VII, 533c). Nel Parmenide si parla di una "potenza del discutere" (hê tou dialegesthai dunamis, 135b), che viene universalmente identificata con la dialettica.

Un'altra caratteristica che accomuna i suddetti quattro dialoghi, e che è stata giustamente enfatizzata da Robinson, è che in essi la dialettica ha sempre a che fare con delle ipotesi (hupotheseis). Nel Menone si dice infatti che, quando non si conosce che cos'è un oggetto, per esempio la definizione della virtù, e tuttavia si vuole conoscere una sua qualità, per esempio se la virtù sia insegnabile o no, si deve formulare un'ipotesi, per esempio assumere che la virtù sia insegnabile, e vedere quali conseguenze ne derivano (86e87b). Partire da un'ipotesi significa dunque assumere una tesi, di cui ancora non si sa il valore, e dedurne le conseguenze: evidentemente il tipo di conseguenze che ne deriveranno fornirà delle indicazioni circa il suo valore. Ma questo procedimento, con buona pace di Robinson, è esattamente l'elenchos praticato da Socrate nei dialoghi giovanili.

Nel Fedone Socrate, che è ormai il portavoce di Platone, narra che, subito dopo essersi "rifugiato nei discorsi", in seguito alla delusione provata per il libro di Anassagora, cominciò a praticare un metodo consistente nell'assumere per ipotesi volta per volta un "discorso" (hupothemenos hekastote logon) e nel considerare vere le cose, cioè le proposizioni, che si accordavano (sumphônein) con esso, e false quelle che non vi si accordavano (99 e-100 a). Poco più oltre egli precisa che, per trovare la causa, cioè la ragione, di qualcosa, bisogna formulare un'ipotesi (hupothesis) e quindi esaminare le conseguenze che derivano da essa, per vedere se concordino o discordino tra loro (sumphônein ê diaphônein). Quando poi sia necessario dare ragione (didonai logon), cioè rendere conto, anche dell'ipotesi formulata, si deve procedere nello stesso modo, cioè formulare un'altra ipotesi, la quale stia "più in alto", cioè sia più universale, fino a che si giunga a "qualcosa di sufficiente". Chi agisce in questo modo, conclude Platone, non si confonde con gli "antilogici", cioè coi Sofisti, che mettono in discussione contemporaneamente il principio (archê) e le conseguenze che ne derivano, ma si comporta come chi fa parte dei filosofi (101d-e).

Il procedimento qui illustrato consiste principalmente di due operazioni: la deduzione delle conseguenze di un'ipotesi e la riduzione di questa ad un'ipotesi ad essa superiore, da cui sia deducibile. La prima operazione riproduce la confutazione operata da Socrate: è chiaro, infatti, che qualora le conseguenze che si deducono da un'ipotesi discordino tra loro, cioè si contraddicano, l'ipotesi deve considerarsi falsa. Qualora invece le conseguenze non discordino tra loro, ciò non significa che l'ipotesi da cui derivano sia vera, ma solo che essa è incontraddittoria, cioè possibile. Per vedere se essa è vera, bisogna poterla dedurre da un'ipotesi superiore, il cui valore sia "sufficiente", cioè sia garantito, e che quindi possa fungere da autentico "principio". Il Fedone non dice come si possa accertare la sufficienza, cioè il valore, di tale principio. Esso tuttavia allude chiaramente alla necessità di integrare il procedimento negativo, cioè distruttivo, con un procedimento positivo, cioè costruttivo, capace di condurre alla verità. 
Un passo ulteriore nella chiarificazione di questo procedimento costruttivo è compiuto nella Repubblica. Qui Platone anzitutto distingue la dialettica dalla matematica in base al diverso uso che ciascuna di queste due discipline fa delle ipotesi. Nelle matematiche "l'anima è costretta a cercare partendo da ipotesi (ex hupotheseôn), ma procedendo non verso un principio, bensì verso una conclusione". Nella dialettica, invece, essa procede partendo ugualmente da un'ipotesi, ma facendo il suo cammino fino ad un principio anipotetico (ep'archên anupotheton, VI, 510b). Le matematiche, dunque, non mettono alla prova le ipotesi mediante la confutazione, ma le assumono senz'altro come vere, cioè come se fossero princìi, e si limitano a dedurne le conseguenze. In esse il valore delle conseguenze dipende tutto dal valore delle premesse, ma poiché questo non è giustificato, l'intero procedimento rimane ingiustificato, cioè è solo "ragionamento" (dianoia), non scienza (epistêmê).

Quando invece "il discorso attinge la capacità dialettica" (tou dialegesthai dunamei), esso considera le ipotesi non come princìpi, ma come vere e proprie ipotesi, cioè come "punti di appoggio e di slancio", fino ad arrivare all'anipotetico, cioè al principio del tutto (tou pantos archê), e solo dopo averlo attinto (hapsamenos autês), deducendone le conseguenze, esso giunge alla conclusione (511b). Qui Platone allude al mettere in questione le ipotesi, cioè al cercare di renderne conto, di darne una ragione, e questa ragione in ultima analisi non può essere data che da un principio anipotetico, cioè un principio che non abbia più bisogno a sua volta di una ragione. Probabilmente egli chiama "principio anipotetico" ciò che nel Fedone aveva chiamato "qualcosa di sufficiente". Ora però Platone chiarisce in quale modo si giunge al principio anipotetico, e lo fa nel libro successivo della Repubblica, dove torna a parlare della dialettica.

Egli afferma infatti che "il procedimento dialettico (hê dialektikê methodos), dopo aver distrutto le ipotesi, procede fino al principio stesso, al fine di consolidarsi", ma aggiunge che esso non giunge a conoscere il bene in sé, ossia il principio, se non riesce, "passando attraverso tutte le confutazioni (dia pantôn elenchôn diexiôn), come in battaglia, e sforzandosi di confutare (elenchein) non secondo opinione, ma secondo realtà", ad aprirsi la strada con un argomento appropriato in tutti questi casi (en pasi toutois)" (VII, 533c534c). Qui Platone non solo indica il procedimento della dialettica nella confutazione - la quale, applicata alle ipotesi, le '"distrugge", cioè le riduce a contraddizione - e dunque ribadisce il carattere confutatorio della dialettica; ma egli precisa ben due volte che è necessario eseguire "tutte" le confutazioni. Qui sta il segreto del carattere costruttivo, non semplicemente distruttivo, della dialettica, e quindi la differenza tra dialettica platonica e quella socratica. Solo, infatti, dopo avere confutato tutte le ipotesi possibili, relative a una determinata questione - nella fattispecie tutte le possibili definizioni del bene -, si ha la sicurezza che l'ipotesi che rimane, ossia che resiste alle confutazioni, è vera, cioè non è più una semplice ipotesi, ma è un autentico principio, il principio appunto anipotetico. Ora, restando ferma la differenza sopra indicata, non c'è dubbio che il tipo di argomentazione di cui la dialettica si serve per distruggere le ipotesi e attingere il principio è, ancora una volta, la confutazione.

La stessa concezione della dialettica si ritrova nel Parmenide. Questo dialogo, infatti, pone nuovamente la dialettica in rapporto con le ipotesi e mostra concretamente che cosa significa "passare attraverso tutte le confutazioni", cioè offre un'illustrazione ampia ed esauriente del procedimento che nella Repubblica è soltanto menzionato. Esso applica infatti il procedimento in questione all'"uno", il quale coincide con quello che nella Repubblica era il bene, cioè il principio anipotetico, e si riallaccia esplicitamente alla dialettica di Zenone, cioè alla dimostrazione di una tesi mediante la confutazione, cioè la riduzione a contraddizione, delle ipotesi ad essa opposte. Nel famoso "intermezzo" del dialogo, dopo che il personaggio di Parmenide ha sottoposto a critica la dottrina delle idee, egli stesso indica a Socrate lo strumento per difenderla nell'"esercizio" (gumnasia) praticato da Zenone, applicato però non più alle cose sensibili, ma alle realtà intelligibili: è l'indicazione esplicita che la dialettica platonica consiste nell'unire il metodo di Zenone con quello di Socrate.

Ma anche la dialettica socratica, puramente distruttiva, viene superata mediante la seguente indicazione messa in bocca a Parmenide: "bisogna però fare un'altra cosa in più, cioè non solo, dopo aver posto come ipotesi (hupotithemenon) l'esistenza di ciascuna cosa, cercare le conseguenze che scaturiscono dall'ipotesi, ma anche, se ti vuoi esercitare meglio, vedere quali sono le conseguenze di un'ipotesi che neghi l'esistenza dell'oggetto della prima" (135e-136a). Dagli esempi che successivamente Parmenide porta risulta che, per ciascuna coppia di opposti (l'uno e i molti, la somiglianza e la dissomiglianza, il moto e la quiete, l'essere e il non essere), si devono anzitutto formulare due ipotesi, l'una positiva e l'altra negativa, relativamente all'esistenza di ciascuno dei due opposti o al suo possesso di una determinata proprietà, e poi vedere quali conseguenze derivano, sia dall'ipotesi affermativa che da quella negativa, per ciascun opposto considerato in se stesso o considerato in rapporto all'altro. Si ottengono così otto tipi di conseguenze, a proposito di ciascuno dei quali si dovrà valutare se esse sono accettabili o inaccettabili, e sulla base di questo risultato si potrà deciedere quale ipotesi, ed in quale senso considerata, è vera, e quale, ed in quale senso considerata, è falsa. Ma questo non è altro che il passare attraverso "tutte" le confutazioni, di cui si parlava nella Repubblica.

Non è questo il luogo per esaminare la complessa struttura del Parmenide, tuttavia è possibile dimostrare che essa corrisponde perfettamente allo schema delineato nell'"intermezzo", e che degli otto tipi di conseguenze che vengono complessivamente dedotti dalle due ipotesi "se l'uno è" e "se l'uno non è", cioè quattro da ciascuna delle due, di cui due concernenti l'uno (considerato in se stesso e considerato in rapporto ai molti) e due concernenti i molti (considerati in se stessi e considerati in rapporto all'uno), alcuni sono ritenuti accettabili ed altri sono ritenuti inaccettabili: sono accettabili tutte quelle conseguenze che mantengono il rapporto reciproco tra l'uno e i molti, ed inaccettabili tute quelle che ne prescindono. In tal modo, malgrado le apparenze di inconclusività dovute alla struttura estremamente complessa dell'argomentazione, si perviene ad una conclusione positiva, cioè che l'uno deve essere sempre considerato in rapporto ai molti e i molti devono essere sempre considerati in rapporto all'uno, ma il procedimento 
attraverso il quale vi si perviene è la dimostrazione della inaccettabilità di tutte le ipotesi ad essa opposte, cioè la confutazione di "tutte" le ipotesi, menzionata nella Repubblica (per questa dimostrazione mi permetto di rinviare al mio saggio Conseguenze inaccettabili e conseguenze accettabili delle ipotesi del "Parmenide", in Istituto Suor Orsola Benincasa, Il "Parmenide" di Platone, a cura di V. Vitiello, Napoli 1992, pp. 47-74).

\section{Divisione e confutazione nei dialoghi della vecchiaia}

Negli ultimi dialoghi in cui Platone parla della dialettica, cioè Fedro, Sofista, Politico e Filebo, quest'ultima non è più messa in connessione con le ipotesi, ma piuttosto con i due procedimenti caratteristici del "riunire" (sunagôgê) e del "dividere" (diairesis) le idee. Ciò tuttavia non costituisce, come vedremo, un abbandono della concezione della dialettica come confutazione, la quale viene anzi esplicitamente ribadita sia nel Sofista sia nella Lettera VII (opera che, chiunque ne sia l'autore, è posteriore ai dialoghi e riflette l'ultima fase del pensiero di Platone). Si tratta piuttosto di una presa d'atto, da parte di Platone, della forza della dialettica, raggiunta mediante l'uso costruttivo della confutazione, e quindi della sua capacità di decidere quali idee debbano essere riunite e quali divise, ossia di affermare e negare secondo verità, come si addice ad una vera e propria scienza.

Nel Fedro, come è noto, l'attività di dividere e riunire (tôn diaireseôn kai sunagôgôn) è esplicitamente considerata compito dei "dialettici" (dialektikoi, 265d-e). Nel Sofista lo straniero afferma che il compito proprio della "scienza dialettica" (dialektikê epistêmê) consiste nel "dividere per generi (to kata genê diaireisthai) e non credere che una specie che è identica sia invece diversa, né pensarla identica se invece è diversa" (253d). La dialettica viene così ad essere l'arte di stabilire quali specie comunicano e quali non comunicano tra di loro, cioè quali affermazione e negazioni sono vere e quali invece sono false: il discorso vero, infatti - precisa in seguito Platone - è quello che unisce o divide fra loro nomi e verbi a seconda che le specie da essi indicate siano rispettivamente unite o divise nella realtà (per esempio "Teeteto è seduto"), mentre quello falso unisce o divide nomi e verbi corrispondenti a specie che non sono rispettivamente unite o divise nella realtà (per esempio "Teeteto vola", 259a-264b). La divisione è dunque il metodo che permette di discernere il vero dal falso.

Nel Sofista e nel Politico, come è noto, la divisione praticata al fine di definire un'idea (l'idea, per esempio, del "sofista", o quella del "politico") è la divisione dicotomica, in cui ad ogni passo si deve scegliere fra due idee opposte, da associare all'idea che si vuole definire. Mediante la scelta dell'una o dell'altra fra tali idee opposte, si decide di considerare vera l'una o l'altra tra due asserzioni contraddittorie, cioè tali da esaurire tutte le possibilità. Che cosa permette di prendere questa decisione? Evidentemente un processo di eliminazione, che non può non far pensare alla confutazione. Se questo è vero, abbiamo ancora a che fare con una dialettica del tipo di quella descritta nellaRepubblica e nel Parmenide.

Nel Filebo, riproponendo la concezione della dialettica come divisione, Platone precisa che questa non deve essere necessariamente dicotomica, ma può anche dar luogo, a seconda dei casi, ad un numero maggiore di distinzioni. Dopo avere infatti stabilito che tutte le idee, in quanto costituite da unità e molteplicità, contengono in sé il limite e l'illimitato, egli afferma: "Di conseguenza, essendo appunto le cose ordinate così, noi dobbiamo sempre ammettere e sempre ricercare ovunque una nota caratteristica unitaria (mian idean $), . .$. e come allora l'abbiamo colta, ... bisogna cercarne ed esaminarne, dopo e subordinatamente alla prima, altre due, se in qualche modo ce ne sono due, e se no tre o un qualche altro numero e poi rifare l'operazione per ciascuna di queste ultime unità, finché la prima unità non solo si vede che è unità e pure molteplicità e anzi infinita molteplicità, ma si vede anche la sua struttura numerica. Non bisogna riferire il carattere di infinità alla molteplicità prima che si sia vista tutta la complessiva struttura numerica di questa, struttura che sta nell'intervallo tra la sua unità e la sua infinità". La determinazione di questo numero esatto, compreso tra l'unità e l'infinità, è ciò che distingue il modo dialettico (dialektikôs) da quello eristico di condurre i discorsi (16c-17a).

Secondo la maggior parte degli interpreti qui Platone allude alla divisione ed afferma la necessità che essa non sia sempre dicotomica, ma determini il numero effettivo delle altre idee comprese in ciascuna idea, cioè delle idee con cui ciascuna idea comunica, il quale può essere superiore a due, ma è sempre un numero finito. Il buon dialettico è colui che determina questo numero con esattezza, ossia colui che sa esattamente con quante idee ciascuna idea comunica. Il senso di questo sapere non può che essere quello di essere sicuri di avere esplorato effettivamente tutte le possibilità, tutte le ipotesi di comunicazione, avendo stabilito non delle alternative in cui uno dei due corni sia determinato e l'altro rimanga indeterminato, come nel caso della divisione dicotomica, ma delle alternative in cui l'intera gamma delle possibilità da scegliersi sia rigorosamente determinata. Mi sembra evidente che, anche in questo caso, il procedimento per mezzo del quale si può compiere una scelta è la confutazione, quindi ancora una volta la dialettica viene ad essere un passaggio attraverso "tutte" le confutazioni.

Ciò è confermato dall'importanza che la confutazione assume nella stessa definizione del sofista, che poi risulta essere anche la definizione del filosofo, cioè del dialettico. È noto, infatti, che nel Sofista l'arte sofistica è definita prima come una forma di caccia, ossia di arte acquisitiva; poi come arte acquisitiva mediante scambio; poi come scambio, cioè commercio, di virtù, cioè di capacità; poi come commercio dell'arte di disputare con i discorsi; infine come commercio dell'arte di dividere (diairetikê), ovvero di separare, i discorsi validi da quelli non validi. Quest'ultima arte viene successivamente precisata come arte di purificare l'anima, che vuol dire di insegnare, anzi di insegnare educando, infine come arte di educare confutando (cioè per mezzo dell' elenchos, 221c229c). La confutazione è infatti necessaria per eliminare quell'ignoranza che è il credere di sapere senza sapere. Essa consiste precisa Platone - nell'interrogare gli uomini che credono di sapere e nel sottoporre ad esame (exetazein) le loro opinioni, mostrando che esse sono in contraddizione fra loro (230b). 
A questo punto Platone si rende conto di avere definito in tal modo non il sofista, ma il dialettico, cioè il filosofo, ed afferma che questa, così definita, è una "sofistica nobile" (gennaia sophistikê). Tra il sofista e il dialettico, del resto, c'è grande somiglianza, perché anche il sofista possiede l'arte del contraddire: la differenza è che il sofista contraddice tutto, perché crede di sapere tutto, mentre il filosofo sa che sapere tutto è possibile solo agli dèi; dunque la sofistica è solo una sapienza apparente, un'imitazione della sapienza (231a-234b). Non c'è dubbio che la rappresentazione qui data della dialettica è quella della dialettica socratica, cioè l'arte del dialogare, dell'interrogare, dell'esaminare e del confutare. Ma Platone la identifica esplicitamente con l'arte del praticare la divisione, afferma che il dividere consiste nel discriminare i discorsi veri da quelli falsi, e dice che per fare questo è necessaria la confutazione. Abbiamo dunque la prova conclusiva dell'identità tra la dialettica diairetica e la dialettica confutatoria.

Tutto questo è confermato dal famoso passo della Lettera VII, dove si afferma che, solo "se si confuta in confutazioni benevole (en eumenesin elenchois elenchomena), facendo uso senza ostilità di domande e risposte, brillano la comprensione e l'intelligenza intorno a ciascuna cosa" (344 b). Si tratta, ancora una volta, della dialettica socratica, che Platone, o un suo discepolo, rievoca alla fine della sua vita, confermando di non averla mai rinnegata, ma di averla invece rafforzata e resa capace di conoscere la verità. Ma ciò è confermato anche da Aristotele, il quale, dovendo indicare la differenza tra la dialettica di Socrate e quella di Platone, afferma che "allora (tote, cioè al tempo di Socrate) non c'era ancora la forza dialettica (dialektikê ischus) tale da potere indagare gli opposti anche indipendentemente dall'essenza e da stabilire se la scienza degli opposti è la stessa" (Metaph. M 4, 1078 b 25-27). L'indagine degli opposti, che conduce ad avere scienza della verità dell'uno attraverso la scoperta della falsità dell'altro, non può che essere la confutazione "forte", cioè quella che elimina tutte le altre possibilità.

bibiographie

BERTI, E., 'Conseguenze inaccettabili e conseguenze accettabili delle ipotesi del "Parmenide"', in Istituto Suor Orsola Benincasa, Il "Parmenide" di Platone, a cura di V. Vitiello, Napoli, 1992.

BERTI, E., Contraddizione e dialettica negli antiche e nei moderni, Palermo, 1987.

BRANDWOOD, L., A Word Index to Plato, Leeds, 1976.

RoBinson, R., Plato's Earlier Dialectic, 2nd edn. Oxford, 1953.

STENZEL, J., Studien zur Entwicklung der platonischen Dialektik von Sokrates zu Aristoteles (2. erw. Aufl. Leipzig und Berlin, 1931). 